Books, videos, CD-ROMs, DVDs and any

other relevant items submitted for a review

in the $B D J$ should be addressed to: Kate

Maynard, Assistant Editor, British Dental

Journal, NPG, 4-6 Crinan Street, London,

N1 9XW

\section{Perio pictures (CD-ROM)}

P. Ower

Ł25 inc p\&tp see www.periocourses.co.uk

This patient education CD-ROM is produced by Phil Ower, who with Graham Smart has a sterling record in postgraduate education. It is Windows-based with all necessary viewing software included in the disc. There are over 430 clinical images and videos, divided into 15 easily navigable sections, explaining periodontal concepts and therapies, ranging from drug-induced gingival overgrowth, perio-endo problems and surgical and non-surgical therapy, to masking gingival recession.

For successful periodontal therapy, patient compliance is essential and anything that can educate the patient must help towards this. Relevant pictures shown to patients would enable these conditions to be explained quickly and succinctly. These pictures are meant to be used by suitable team members who have first familiarised themselves with the help of the clear, concise and comprehensive accompanying notes. The slides themselves have minimal words to avoid detracting from the power of the image. I was initially sceptical of this but soon became convinced.

If a picture 'speaks a thousand words' then the images on the video clips of the use of the single tufted brush speak volumes. After watching these, I was shocked to find that I was not using these useful tools correctly nor was my method of oral hygiene instruction - by demonstrating in the patient's mouth at the wall mirror - ideal. Patients cannot always see their own mouths in a mirror, as well as moving pictures on a screen. Therefore, this must be an essential preliminary before 'feeling' it in their own mouths.

The results of self-performed plaque control alone are well illustrated. Resisting the immediate urge to start removing the calculus and allowing the patients to see the improvement with only plaque control must be a powerful form of motivation, enhancing their sense of self-sufficiency. This also reinforces the concept that it is plaque (the patient's responsibility!) and not calculus that causes the disease.

It is impossible to have pictures for every condition but I would have liked some images of severe periodontal breakdown from smoking to show to my persistent smokers, to shock them into giving up!

As most practices do have computers, this inexpensive CD should form an integral part of their educational material. I thoroughly recommend it!

R. Ladwa

\section{Implant restorations: a step-by-step guide (second edition)}

\section{Drago \\ UK: Blackwell Munksgaard \\ price 555.00 ; pp 264 \\ ISBN 9780813828831}

Text is split into three sections. The first section relates to the technical components of the $3 \mathrm{i}$ system, diagnosis and treatment planning. The second section describes the management of several clinical cases ranging from basic to the more demanding. The final section discusses record-keeping, patient compliance, hygiene regimes and follow-up. The last chapter, somewhat oddly, considers osseointegration, which may have been more useful earlier in the book.

The text is highly illustrated with good quality images and tables and follows a format of demonstrating how to treat common treatment scenarios and problems.

The text is easy to read and provides a high level of detail. The strengths and weaknesses of this text are exemplified by the fact that it relates only to $3 \mathrm{i}$ products and in such detail that it is almost a manual for this system. This is obviously of great advantage if you are a $3 i$ user but can be frustrating if you are not. This latter feature can detract from the many positive features of this publication.

This book will be of great use to those using the $3 \mathrm{i}$ system but its focus on this system may put off those who are not.

K. Bishop

\section{Concise encyclopedia of periodontology}

\author{
D. C. Vandersall \\ UK: Blackwell Munksgaard \\ price £39.99; pp 232 \\ ISBN 9780813826028
}

In essence, this book is a collection of key periodontal terms listed in alphabetical sequence. Each entry has a short definition with appropriate references and there are several good quality photographs to illustrate the text. There is an appendix of influential authors that have contributed to the periodontal literature and a list of key references to accompany the text. The title Concise encyclopedia... is something of an oxymoron, although one has to admire the author's attempt at presenting such a large amount of information in a relatively small book.

The book aims to provide a 'concise, succinct, and portable adjunct to traditional textbooks'. The publishers state that it enables readers to search the literature by cross-referencing terms to classic and modern sources and to review specific areas of the subject. There is no doubt that the book contains some very useful information, but I would have preferred this to have been presented in a more traditional format, with separate chapters for each subject rather than an alphabetical list of terms. This would have been more useful for postgraduate students preparing for examinations (the main target audience of this book).

I am particularly pleased to see that every entry in the main body of the text has at least one supporting reference. This approach is quite unusual but extremely useful for any student of periodontology. The aim is to allow readers to 

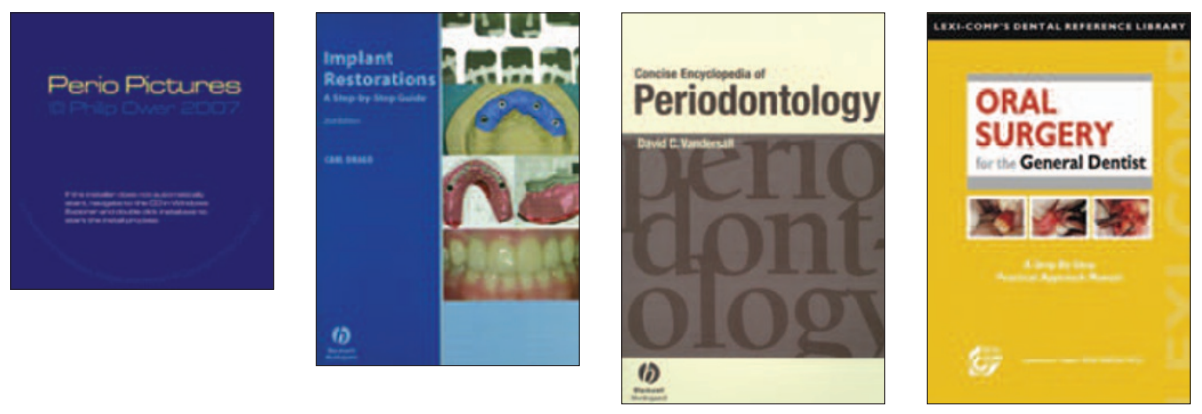

cross-reference specific terms to classical papers and their authors. Unfortunately, the book is in danger of becoming outof-date quite rapidly as important new references become published in the scientific press. In this era of evidence-based dentistry, systematic reviews and other high quality studies are becoming more and more relevant to support everyday clinical practice. It is difficult to see how a book of this nature can keep pace with the volume of papers that are published in the core periodontal journals.

Some of the terms are extremely archaic and are included only for historical interest eg Balneotherapy or Schmutz Pyorrhea! American terminology and spellings are used throughout, and I suspect that a larger proportion of the key references are drawn from the North American literature rather than European publications. This may make the book a little less useful to readers from other countries, including the UK.

Nevertheless, this publication remains a useful source of information.

G. Sharpe

\section{Oral surgery for the general dentist}

\section{I. Gaum}

USA: Lexi-Comp

price $\$ 54.95$; pp 170

ISBN 1591951690

This is a well laid out manual with a thorough introduction followed by chapters on the various routine surgical procedures and aspects of patient care. The book concludes with helpful hints.

Oral surgery cannot be taught with words alone, and hence any oral surgery book of consequence should rely heavily on illustrations and photos. By its nature, photographs of surgical procedures are difficult to follow due to orientation and a field covered with blood. This book tackles this difficulty by having excellent clinical photos almost always beside a photo of a prosthetic oral cavity displaying the procedure (without excess blood). The scope of the book is ideal for the general practitioner as routine surgical procedures are described in detail (such as surgical removal of roots) allowing the practitioner to broaden their skill base. However, the book is not suitable as a lone oral surgery book for the undergraduate as much of the very fundamental aspects of oral surgery technique are omitted, such as how to use elevators and why.

Oral surgery should always be a planned procedure; however procedures do not always go to plan. Being able to deal effectively with complications will greatly reduce stress, and the book highlights many tips to reduce time and frustration. Examples include the ART (Anterior Ramus Technique - the author is the principal developer) procedure for inferior dental blocks (although the author says he still uses two cartridges doing the ART procedure so I am unsure as to whether this is a better system, but again having a choice of methods is very useful) and the use of curved forceps to extract lower roots with their initial placement on the mucosa thus facilitating a luxating movement, and finally a crane pick (a cryers type instrument which is inserted into a hole in the tooth drilled by the dentist).

Management of oral surgery patients should include effective post-operative management, and this is well covered with helpful hints such as patients are given monojet syinges and shown how to wash out third molar sockets.

Negatives; well as the author is Canadian there is the expected different naming of common instruments (a couplands being referred to as a gouge), and I feel an up-to-date manual should have a section on implants - or at least stress the importance of bone preservation when performing extractions and show how this can be done.

Overall this is a well laid out manual with clear photos and illustrations; however it may leave you wanting a crane pick! 